\title{
Viewpoint
}

\section{Untested natural medicines are big threats to health in China and Africa}

\author{
Minghui Zhang ${ }^{1}$ \\ ${ }^{1}$ School of Environmental Science and Engineering, Shandong University, Qingdao, China \\ Keywords: africa, china, natural medicines \\ https://doi.org/10.29392/001c.13651
}

Journal of Global Health Reports

Vol. 4, 2020

\begin{abstract}
In mainland China and Africa, when natural medicines apply for licenses, they do not need to pass the same rigorous toxicity and efficacy tests as those of synthetic medicines. The lack of regulation of untested natural medicines by drug administration departments is a big threat to global health. Studies have shown natural medicines severely threaten the health of human beings although they seem harmless. Over 100 million of the Chinese are suffering from diseases caused by natural medicines. These medicines include aristolochic acid (AA)-containing plants (a worldwide problem) and natural medicine injections (only in mainland China). Clear evidence shows the widespread toxicity of AA-containing plants and natural medicine rejections while not a single rigorous test suggests the efficacy of them. One recent example is that plant soups were widely used to treat patients in mainland China during the coronavirus disease 2019 (COVID-19) outbreak. An ingredient of these plant soups is Xixin, which contains AA.
\end{abstract}

In mainland China and Taiwan, plants and wild animals are widely used as medicines based on traditional medicine books without being noticed that they might contain toxic molecules. For example, 33\% of the Chinese in Taiwan had been prescribed aristolochic acid (AA)-containing medicines. ${ }^{1}$ AA can induce irreversible damage to kidney even with trace amounts. ${ }^{2}$ It is estimated that 100 million of the Chinese (i.e. 7\%) in mainland China suffer from kidney diseases caused by AA-containing medicines. ${ }^{3}$ Liver cancers ${ }^{4,5}$ and urothelial cancers ${ }^{6}$ are also associated with AA-containing medicines. By sequencing the whole exomes, it was observed that $25.8-47 \%$ of liver cancers in mainland China ${ }^{4,5}$ might be caused by AA-containing medicines and this number increased to $78 \%$ in Taiwan. ${ }^{5}$ Recently, during the COVID-19 outbreak, National Administration of Traditional Chinese Medicine in mainland China reported that 214 patients with coronavirus recovered after drinking plant soups (named as Qingfeipaidutang) as of 5 February 2020. Based on this report, National Health Commission of China required hospitals to treat patients for coronavirus by using these plant soups. However, this report is lack of randomized controlled trials. What is more terrible is that an ingredient of these plant soups is Xixin, which contains AA, unfortunately. ${ }^{7}$ AA-containing medicines or soups are widely used because (1) traditional Chinese medicine books recorded that these medicines can be used to treat diseases including lung diseases, (2) the toxicity of AA is chronic so that patients cannot feel the toxicity immediately, and (3) randomized controlled trials were not performed rigorously so that the efficacy was misinterpreted.

Natural medicine injections are widely used in mainland China but are not studied to the same degree as that of oral medicines. The sales of natural medicine injections were over CN¥100 billion in 2018 although adverse drug reactions were reported frequently. ${ }^{8}$ Extracted compounds from plants or animals are a mixture of complex molecules and the molecular structures are not identified. It is incredible that rigorous animal experiments and clinical trials have never been carried out for these natural medicine injections. One recent example was that natural medicine injections (e.g. Xuebijing, Tanreqing) were recommended by National Health Commission of China to treat patients for coronavirus. Another example was that when newly born babies had jaundice, some doctors in mainland China would inject Yinzhihuang into the blood of babies. Some babies died after injection because of allergy to extracts or fever induced by endotoxins of contaminated bacteria during manufacture. The good news is that Yinzhihuang injection has been forbidden by China Food and Drug Administration (which was named as National Medical Products Administration in April 2018) since October 2016. As of December 2017, however, 134 kinds of natural medicine injections from 224 pharmacy companies were still given the licenses and produced in mainland China. ${ }^{9}$ China Food and Drug Administration failed to forbid all of these natural medicine injections because (1) not every person is allergic to injections and not every injection has endotoxins so that it is not straightforward to observe the toxic effect, and (2) the government supports natural medicine injections. In 1940, Xinzhong Qian, who was the minister of health, invented the first natural medicine injection Chaihu. The government is proud of this invention and has supported such kind of natural medicine injections since 1940 until now.

\section{THE UNITED STATES}

In U.S., although some natural medicines cannot get the licenses, they are used as alternative medicines or complementary medicines. ${ }^{10}$ The good news is that the toxicity of these natural medicines has been tested. ${ }^{10}$ Namely, these 
alternative natural medicines are harmless. However, the efficacy of these alternative natural medicines is unknown.

If natural medicines want to get licenses from U.S. Food and Drug Administration, they need to pass equally rigorous toxicity and efficacy tests as those of synthetic medicines. Before 2004, the molecular structure of natural medicines has to be identified; since 2004, the molecular structure does not need to be identified. But the toxicity and efficacy tests are always necessary for natural medicines. Some Chinese pharmacy companies would like to sell natural medicines to U.S., but none of the natural medicines from mainland China has passed the toxicity and efficacy tests of U.S. Food and Drug administration.

\section{AFRICA}

Unfortunately, in Africa, natural medicines are widely used due to its own historic reasons and mainland China's impact as well.

$80 \%$ of the African (i.e. 972 million) regularly use natural medicines. ${ }^{11}$ The license for natural medicines is either unnecessary or easy to obtain in Africa. For example, in Ghana, Center for Plant Medicine Research was established by the Government of Ghana, which can give licenses for natural medicines. It takes $\$ 250$ to analyze herbal products for the purpose of the registration by the Food and Drugs Authority. As the test fee is only $\$ 250$, I suppose that the test is preliminary without using the rigorous tests as those of synthetic medicines.

China has sent natural medicine practitioners to Africa since the 1960s. Thousands of African students have studied natural medicines and traditional medicine practices in mainland China and tens of medical centers for natural medicines have been established in Africa since 2000. Given the widespread toxicity of AA-containing natural medicines in mainland China, ${ }^{2,6}$ Africa might be threatened by AAcontaining medicines as well and this needs study. In addition, acupuncture is introduced to Africa to treat patients for human immunodeficiency viruses (HIV). The needles used for acupuncture might transmit HIV to other patients and this needs study.

Wild animals are transported from Africa to mainland China. Animals such as pangolins in Africa are killed and transported due to the huge market of mainland China. ${ }^{12}$ Doctors specializing in traditional Chinese medicines believe that the scale of pangolins can be used to treat certain human diseases because traditional Chinese medicine books recorded so. However, not a single rigorous experiment suggests its efficacy and identifies the effective molecules inside the scale of pangolins. Pangolins are already critically endangered in mainland China. If the illegal killing and transport continue, pangolins might become critically endangered in Africa as well.

\section{CONCLUSIONS}

In order to protect the large population and wild animals, the drug regulation departments need to carry out the following measures. (1) AA-containing medicines should be forbidden immediately. In 1993, it was warned that some plants induced rapidly progressive kidney fibrosis, which was ascribed to AA later ${ }^{2}$ since 2003 , AA-containing medicines have been forbidden in Taiwan; in 2006, it was warned that Xixin contained AA $;{ }^{7}$ in 2020 , Xixin was still used as an ingredient of plant soups to treat patients for coronavirus in mainland China. (2) All of the natural medicine injections should be forbidden immediately. Not a single rigorous test suggests the efficacy of them. But the risk of death exists because of allergy to extracts or fever induced by endotoxins of contaminated bacteria. (3) When natural medicines apply for licenses, they need to pass equally rigorous efficacy and toxicity tests as those of synthetic medicines. The processes and results of tests need be uploaded to the internet and need to be open to the public to prevent corruption. In 2007, Xiaoyu Zheng, who was the minister of China Food and Drug Administration, was sentenced to death. The reason is that he got some bribe money in exchange for licenses to medicines and some patients died from these medicines. Regulations need to be made clear and processes need to be transparent to prevent such kind of corruption. (4) Natural medicines can be re-evaluated and the licenses can be retracted when severe toxicity is observed. (5) The killing and torturing of wild animals need to be forbidden. Although ursodiol inside bear pile has certain efficacy in treating human liver diseases, ursodiol can be synthesized without torturing bears since the 1950s. In 2020 in mainland China, bear pile is still isolated from bear's gallbladder cruelly when bear is alive. (6) Apart from AA-containing plants, other toxic plants need to be forbidden to be consumed as medicines (e.g. pyrrolizidine alkaloids-containing plants) or food (e.g. fish mint eaten as salad in Guizhou Province of China) although they seem harmless.

It is true that some medicines (e.g. artemisinin) ${ }^{13}$ can be extracted from natural plants, animals, or microorganisms. It is worth mentioning that such kind of natural medicines has a single molecular structure and the molecular structure has been identified. More importantly, the toxicity and efficacy have been tested rigorously. However, untested natural medicines should be discouraged by drug regulation departments as the risk far outweighs the benefit. At least, the toxicity of natural medicines should be tested to ensure that they are harmless.

Acknowledgements: The author would like to thank Dr. Benjamin Bandowe for contributing to the codiscussions.

Funding: No specific funding was received for this study.

Author contributions: MZ had the idea, conducted investigation, and wrote the manuscript

Competing interests: The author completed the Unified Competing Interest form at www.icmje.org/coi disclosure.pdf (available upon request from the corresponding author), and declare no conflicts of interest.

\section{Correspondence to:}

Minghui Zhang 
School of Environmental Science and Engineering Shandong University, Qingdao, China

mhzhang1005@gmail.com

This is an open-access article distributed under the terms of the Creative Commons Attribution 4.0 International License (CCBY-4.0). View this license's legal deed at http://creativecommons.org/licenses/by/4.0 and legal code at http://creativecommons.org/licenses/by/4.0/legalcode for more information. 


\section{REFERENCES}

1. Hsieh S-C, Lin I-H, Tseng W-L, Lee C-H, Wang J-D, et al. Prescription profile of potentially aristolochic acid containing Chinese herbal products: An analysis of National Health Insurance data in Taiwan between 1997 and 2003. Chinese Medicine. 2008;3(1):13. doi:1 $\underline{0.1186 / 1749-8546-3-13}$

2. Debelle FD, Vanherweghem JL, Nortier JL.

Aristolochic acid nephropathy: A worldwide problem. Kidney international. 2008;74(2):158-169. doi:10.103 8/ki.2008.129

3. Grollman AP. Aristolochic acid nephropathy: Harbinger of a global latrogenic disease. Environ $\mathrm{Mol}$ Mutagen. 2013;54(1):1-7. doi:10.1002/em.21756

4. Lu Z-N, Luo Q, Zhao L-N, Shi Y, Wang N, et al. The mutational features of aristolochic acid-induced mouse and human liver cancers. Hepatology. 2019. do i:10.1101/507301

5. Ng AWT, Poon SL, Huang MN, et al. Aristolochic acids and their derivatives are widely implicated in liver cancers in Taiwan and throughout Asia. Sci Transl Med. 2017;9(412):6446. doi:10.1126/scitranslm ed.aan6446

6. Chen CH, Dickman KG, Moriya M, Zavadil J, Sidorenko VS, et al. Aristolochic acid-associated urothelial caner in Taiwan. P Natl Acad Sci USA. 2012;109(21):8241-8246. doi:10.1073/pnas.11199201 $\underline{09}$
7. Yang YL, Lin JL, Chen KH, Yu CC, Hsu PY, et al. Aristolochic acid-related nephropathy associated with the popular Chinese herb Xi Xin. J Nephrology. 2006;19(1):111-114.

8. Wang L, Yuan Q, Marshall G, Cui X, Cheng L, et al. Adverse drug reactions and adverse events of 33 varieties of traditional Chinese medicine injections on National Essential Medicines List (2004 edition)of China: an overview on published literatures. J Evid Med. 2010;3(2):95-104.

9. Li H, Wang S, Yue Z, Ren X, Xia J, et al. Traditional Chinese herbal injection: Current status and future perspectives. Fitoterapia. 2018;129:249-256. doi:10.1 016/i.fitote.2018.07.009

10. Angell M, Kassirer JP. Alternative medicine - The risks of untested and unregulated remedies. $N$ Engl J Med. 1998;339(12):839-841. doi:10.1056/neim199809 $\underline{173391210}$

11. Bodeker G, Kronenberg F. A public health agenda for traditional, complementary, and alternative medicine. Am J Public Health. 2002;92(10):1582-1591.

12. Xu L, Guan J, Lau W, Xiao Y. An overview of pangolin trade in China. TRAFFIC September. 2016:1-10.

13. Tu Y. The discovery of artemisinin (qinghaosu) and gifts from Chinese medicine. Nature medicine. 2011;17(10):1217-1220. doi:10.1038/nm.2471 